\title{
Low-energy tetrahedral polymorphs of carbon, silicon, and germanium
}

\author{
Andrés Mujica \\ Departamento de Física, MALTA Consolíder Team, \\ Universidad de La Laguna, La Laguna 38206, Tenerife, Spain \\ Chris J. Pickard \\ Department of Physics \& Astronomy, University College London, \\ Gower Street, London WC1E 6BT, United Kingdom \\ Richard J. Needs \\ Theory of Condensed Matter Group, Cavendish Laboratory, \\ J J Thomson Avenue, Cambridge CB3 OHE, United Kingdom
}

(Dated: March 11, 2015)

\begin{abstract}
Searches for low-energy tetrahedral polymorphs of carbon and silicon have been performed using density functional theory computations and the ab initio random structure searching (AIRSS) approach. Several of the hypothetical phases obtained in our searches have enthalpies that are lower or comparable to those of other polymorphs of group 14 elements that have either been experimentally synthesized or recently proposed as the structure of unknown phases obtained in experiments, and should thus be considered as particularly interesting candidates. A structure of Pbam symmetry with 24 atoms in the unit cell was found to be a low energy, low-density metastable polymorph in carbon, silicon, and germanium. In silicon, Pbam is found to have a direct band gap at the zone center with an estimated value of $1.4 \mathrm{eV}$, which suggests applications as a photovoltaic material. We have also found a low-energy chiral framework structure of $P 4_{1} 2_{1} 2$ symmetry with 20 atoms per cell containing fivefold spirals of atoms, whose projected topology is that of the so-called Cairo-type twodimensional pentagonal tiling. We suggest that $\mathrm{P} 4_{1} 2_{1} 2$ is a likely candidate for the structure of the unknown phase XIII of silicon. We discuss Pbam and $\mathrm{P} 4_{1} 2_{1} 2$ in detail, contrasting their energetics and structures with those of other group 14 elements, particularly the recently proposed $\mathrm{P} 4_{2} / \mathrm{ncm}$ structure, for which we also provide a detailed interpretation as a network of tilted diamond-like tetrahedra.
\end{abstract}

PACS numbers: 71.15.Nc,62.50.-p,61.50.-f,61.66.-f

\section{INTRODUCTION}

The group 14 elements carbon (C), silicon ( $\mathrm{Si}$ ), and germanium $(\mathrm{Ge})$ have attracted much interest and have been extensively studied. These elements have an $s^{2} p^{2}$ valence electronic configuration, which leads to common chemical features, but also significant differences. Pure $\mathrm{C}$ is found on the Earth mainly in the graphite and cubic diamond (cd) forms, which exhibit some of the strongest bonds known in Nature. Pure Si and Ge also adopt the diamond structure under ambient conditions, with ideal tetrahedral coordination, and they are, of course, semiconductors of great importance in the electronics industry. Under applied pressure of around $11 \mathrm{GPa}$ both $\mathrm{Si}$ and Ge transform into the $\beta$-Sn-type structure, ${ }^{1}$ which has $4+2$ coordination and is metallic. Other stable and well researched phases of these elements exist at higher pressures. $^{1,2}$

Besides their thermodynamically stable forms, a number of zero-pressure metastable polymorphs have been observed, with both higher and lower densities than the corresponding diamond phases. The lonsdaleite or hexagonal diamond form of carbon, which has a density similar to cubic diamond, was first observed in meteorite craters ${ }^{3}$ and later synthesized from graphite under high pressure and temperature, ${ }^{4}$ and also by chemical vapor deposition and other chemical methods. Hexagonal diamond has also been reported in Si and Ge after decompression and heating from the high-pressure phases or after indentation. ${ }^{5-7}$ Among the high-density polymorphs, the cubic bc8 phase of $\mathrm{Si}$ and tetragonal st12 phase of Ge were first obtained about fifty years ago as zero-pressure tetrahedral metastable forms recovered upon decompression from the respective high-pressure $\beta$ Sn-type phases. ${ }^{5,8,9}$ The $\mathrm{r} 8$ structure (a rhombohedral distortion of the bc8 structure) was later discovered experimentally in high-pressure experiments on quenched $\mathrm{Si}^{10}$ and has recently been identified in nanoindented $\mathrm{Ge}$ samples. ${ }^{11}$ Although the bc8 structure has also been reported in Ge, ${ }^{12,13}$ the st12 structure has not so far been observed in Si. ${ }^{14}$ Low density open framework clathratetype structures of $\mathrm{Si}$ and Ge, with several potential applications, can also be produced at ambient pressure using chemical synthesis methods and have been the object of intense research in recent years. ${ }^{15-18}$ There are also several reports of other $\mathrm{Si}$ and Ge phases obtained upon pressure release in diamond anvil cells or by nanoindentation and whose structure has not yet been experimentally resolved. ${ }^{6,7,19-22}$

Carbon, with the versatility that stems from its unique ability to form $s p, s p^{2}$, and $s p^{3}$ hybrid bonds, provides most examples of allotropes with a wide range 
of structures and properties. These allotropes include, besides those already mentioned, fullerenes, nanotubes, and graphene and its close relatives. ${ }^{23}$ New structural forms have been added to this list in recent times. A low-temperature quenchable transparent and superhard metastable crystal phase of carbon, for which several structures have been proposed, can be obtained by cold compression of graphite, ${ }^{24}$ whereas the application of pressure under different controlled conditions to fullerenes and carbon nanotubes leads to the formation of some new and elusive forms. ${ }^{25-27}$ These findings have excited the imagination and attracted the attention of researchers to this field and it is expected that new experimental observations will occur in the coming years.

Along with experimental investigation of observed allotropes, great effort has been expended in theoretical searches for new tetrahedral phases whose predicted mechanical, structural and optical properties may have potential in advanced technological applications. Such theoretical experiments may result in a fruitful interplay between theory and practice. There is a need for computer simulations that help in characterizing new phases obtained in the laboratory precisely, as such phases are difficult to study in high-pressure experiments, and simulations can significantly help in identifying the correct structure, or in discarding others.

A number of novel hypothetical phases have been proposed from these theoretical studies. One driving force in the search for new carbon phases has been the quest for superhard materials - novel materials with hardness rivalling or even exceeding that of carbon diamond, which could be of technological importance. ${ }^{28-32}$ For silicon, the main material used so far in the fabrication of solar cells, there is interest in new phases with electronic band structures and optical properties better suited than the diamond phase for photovoltaic applications. ${ }^{33,34}$ There is also a purely fundamental interest in researching the possibilities of structural and functional diversity of this important class of elemental materials.

Here we describe a number of hypothetical energetically favourable polymorphs of $\mathrm{C}$, Si, and $\mathrm{Ge}$, including two particular low-energy polymorphs of space group symmetries Pbam and $P 4_{1} 2_{1} 2$. These structures were obtained in density-functional-theory (DFT) based searches. They are somewhat less dense than the corresponding diamond structures and have very similar densities to a low-energy $\mathrm{P} 4_{2} / \mathrm{ncm}$ structure that was recently found in DFT structure searches ${ }^{35}$ and has been considered as a candidate for the observed metastable phase XIII of Si. ${ }^{6,7,20-22}$ The Pbam structure has a somewhat lower energy in $\mathrm{C}, \mathrm{Si}$, and Ge than the $\mathrm{P} 4_{1} 2_{1} 2$ and $\mathrm{P} 4_{2} / \mathrm{ncm}$ structures. It may be hoped that some of these low-energy polymorphs can be synthesized experimentally.

The rest of the paper is organized as follows. In Sec. II we give details of our $a b$ initio calculations and the structural searches performed. In Sec. III we show our results for the materials considered, including detailed descrip- tions of several selected structures. Finally, in Sec. V we present a summary of our most important conclusions. The plentiful data generated during the study has made it necessary to place a portion of them as Supplemental Material to the present paper. ${ }^{36}$

\section{AB INITIO CALCULATIONS AND STRUCTURE SEARCHING}

We performed computational searches for low-energy structures of $\mathrm{C}$ and $\mathrm{Si}$ using first-principles densityfunctional-theory (DFT) methods and the ab initio random structure searching (AIRSS) approach. ${ }^{37,38}$ We did not perform searches for Ge, instead we took the lowenergy Si structures and re-relaxed them for Ge, as lowenergy polymorphs of $\mathrm{Si}$ are also expected to be lowenergy polymorphs of Ge. In the AIRSS approach an ensemble of randomly chosen initial structures are relaxed to a minima of the energy. AIRSS has been successfully employed in finding low-energy structures in many systems, including group 14 elements and their compounds. ${ }^{39-44}$ We performed searches for structures with up to 24 atoms per unit cell.

The CASTEP ${ }^{45}$ DFT code and the Perdew-BurkeErnzerhof $(\mathrm{PBE})^{46}$ Generalized Gradient Approximation (GGA) density functional were employed for the searches. We used ultrasoft pseudopotentials ${ }^{47}$ with the outermost four valence electrons treated explicitly and default basis set energy cutoffs, further relaxing the structures of interest at a higher level of accuracy, using a k-point grid of spacing $2 \pi \times 0.03 \AA^{-1}$ for the Brillouin zone integrations. The details of this procedure are similar to those reported in previous studies. ${ }^{37,38}$

The structures were subsequently re-relaxed using the PBE functional and the Vienna ab initio simulation package (VASP). ${ }^{48,49}$ For Ge we tested pseudopotentials including the four valence electrons explicitly and pseudopotentials in which the $3 d$ electrons of Ge were also included explicitly, but the effects of including the $3 d$ electrons were very small, and we chose to perform phonon calculations with the $d$ electrons treated as core states. Calculations of the energies as a function of volume, phonon spectra, and band structures were performed using the projector augmented-wave (PAW) method. ${ }^{50,51}$ The phonon calculations were performed with the PHON $\operatorname{code}^{52}$. The plane wave cutoff energies used for the VASP calculations were $520 \mathrm{eV}(\mathrm{C}), 320 \mathrm{eV}$ (Si), and $230 \mathrm{eV}$ (Ge, $375 \mathrm{eV}$ for the calculations with the $3 d$ electrons). Dense k-point grids were used for the Brillouin zone integrations (e.g., an $8 \times 8 \times 8$ grid was used for the Pbam structure, with 24 atoms per cell). The structural relaxations were deemed to be converged when all of the forces were less than $5 \mathrm{meV} \AA^{-1}$ and the anisotropy of the stress tensor was less than $0.1 \mathrm{GPa}$. The results of the CASTEP and VASP calculations were in excellent agreement with one another, which is a useful crosscheck. We also performed VAsP calculations using the local spin-density ap- 
proximation (LSDA) ${ }^{53}$ and PBEsol density functionals, ${ }^{54}$ which gave qualitatively very similar results to the PBE calculations and allowed us a full comparison among the three functionals.

We calculated the total energy $E$ as a function of volume $V$ for each phase and fitted the data using a fourthorder Birch-Murnaghan equation of $\operatorname{state}^{55}$ (EoS) from which the pressure, $p$, and the enthalpy, $H=E+p V$, were obtained.

\section{RESULTS}

\section{A. Energetics}

Enthalpy-pressure relations for a selection of different structures in $\mathrm{C}, \mathrm{Si}$, and Ge are shown in Figs. 1, 2, and 3. Calculated equilibrium zero-pressure volumes $V_{0}$, bulk moduli $B_{0}$, and pressure derivatives of the bulk modulus $B_{0}^{\prime}$, obtained from the EoS fitting, are collected in Tables II, III, and IV, for $\mathrm{C}, \mathrm{Si}$, and Ge, respectively. The differences in energy from the corresponding diamond structures at zero pressure are also reported in Tables II-IV. For a given structure, these energy differences generally decrease with increasing atomic number. (See also Supplemental Material. ${ }^{36}$ )

In general, we find good agreement between the calculated and experimental values of structural parameters for the observed phases. For example, our values of the volume, bulk modulus and pressure derivative of the bulk modulus of C-cd are in good agreement with the experimental values of $V_{0}=5.6738(13) \AA^{3}$ (room temperature), ${ }^{56} B_{0}=444(4) \mathrm{GPa}^{57}$ and $B_{0}^{\prime}=3.65(5)$, data from similar DFT calculations, ${ }^{58,59}$ and values from accurate quantum Monte Carlo calculations. ${ }^{60,61} \mathrm{We}$ have adopted the experimental data of Ref. 57 for $B_{0}$ and $B_{0}^{\prime}$ of C-cd, with the revised pressure scale of Ref. 58. Likewise, we obtain good agreement with the experimental data for Si-cd and Ge-cd.

\section{B. Overview of the findings}

It is normally possible to identify families of related crystalline structures. For example, the cd and hd structures are members of an infinite family of low-energy polytypic structures consisting of different stackings of layers. Structures consisting of (1) different stackings of layers; (2) regions of the most stable cd phase and interfacial regions; (3) periodic arrays of point defects in the cd structure etc., can be dreamt up with energies per atom arbitrarily close to that of the corresponding cd structure. It is not our intention to investigate such families of tetrahedrally-bonded group 14 structures, but instead we seek structures which differ in substantial ways from known structures.

Our searches produced many structures, including the observed equilibrium cubic diamond (cd) structure of $\mathrm{C}$ and $\mathrm{Si}$, the hexagonal diamond structure (hd), ${ }^{62}$ and dense structures adopted by $\mathrm{Si}$ under high pressures, such as the $\beta$-Sn structure. As well as finding experimentally observed tetrahedrally-bonded phases we found many structures that have been reported in recent DFT searches of group 14 elements and considered others explicitly for the sake of comparison. ${ }^{29,35,42,64-66}$ (Though we will not report on all of them, some further data can be found in the Supplemental Material. ${ }^{36}$ ) We refer to the new structures by the names of their space groups and use common abbreviations for the well established structures, although some structures will be occasionally also referred to by other names with which they have appeared in the recent literature on this subject. We focus here on tetrahedrally bonded low-energy structures.

\section{High-density tetrahedral polymorphs}

In our searches we found all the dense tetrahedral polymorphs that have been observed in $\mathrm{Si}$ and/or Ge: the bc8 and r8 structures obtained upon decompression from the corresponding high-pressure $\beta$-Sn phase or nanoindentation, ${ }^{5,10-12,67}$ and the st12 structure which has only been observed upon decompression in Ge. ${ }^{8,13,14}$ We also found a body-centred tetragonal structure with space group $I 4_{1} / a$ and 8 atoms per primitive cell, whose enthalpy is remarkably close to that of $\mathrm{r} 8 / \mathrm{bc} 8$, and which plays a role in the decompression kinetics from the high pressure phases. ${ }^{63}$ For Si within the PBE, for example, $\mathrm{I} 4_{1} / \mathrm{a}$ is only $5 \mathrm{meV}$ per atom higher in enthalpy than $\mathrm{bc} 8 / \mathrm{r} 8$ at zero pressure (and about the same enthalpy as st12, within the accuracy of our calculations), see Table III, which makes $\mathrm{I} 4_{1} / \mathrm{a}$ an energetically competitive high-density polymorph.

Also belonging with the group of high-density tetrahedral polymorphs, an hexagonal structure of $P 6_{4} 22$ symmetry labelled cintet is interesting as it is the elemental analog of the tetrahedral variant of the binary cinnabar structure (also called pseudo-cinnabar structure) observed at high pressures in both ZnTe and GaAs. ${ }^{1,2,68}$ At zero pressure, the cintet structure in $\mathrm{Si}$ and Ge is very close in energy to the Ibam structure with 6 atoms per primitive cell recently proposed by Malone and Cohen. ${ }^{64}$ They are both, however, higher in energy than the other observed dense allotropes bc $8 / \mathrm{r} 8 / \mathrm{st} 12$, which may impede their direct synthesis as metastable phases on pressure release from the high-pressure $\beta$-Sn phase. At higher pressures Ibam is lower in enthalpy than cintet. In general, the high-density allotropes have equilibrium energies significantly higher than those of the low-density forms that we discuss next. However, their reduced volumes make them competitive at high pressures, and indeed it is under such conditions that some of them have been synthesised. Among the phases considered, the cintet phase has a bulk modulus second only to the diamond forms of $\mathrm{C}$, and somewhat higher to their respective diamond forms in $\mathrm{Si}$ and Ge. 


\section{Low-energy and lower-density tetrahedral polymorphs}

One interesting result of our search is that we have found a C-centred orthorhombic structure of Cmca symmetry containing 16 atoms in the primitive unit cell whose $E(V)$ curve is almost degenerate, in each of the group 14 elements studied, with that of the Cmmm structure (with 8 atoms per primitive cell) previously found by Zhao et al. ${ }^{30}$ in carbon and named $\mathrm{Cco}_{-} \mathrm{C}_{8}$ by these authors. This Cmmm structure has been proposed as that of a superhard carbon allotrope recovered from room-temperature compression of carbon nanotube (CNT) bundles, ${ }^{27}$ and it is indeed structurally related to the $(2,2) \mathrm{CNT}^{30}$ The Cmmm carbon phase of Zhao et al. was also readily found with AIRSS.

From our calculations, both the Cmmm and Cmca structures are very close in energy to the Pmmn structure of the so-called $P$ carbon phase, with 16 atoms per cell, alternatively proposed by Niu et $a l^{31}$ as the structure of the cold-compressed CNT obtained in experiment in Ref. 27. Both the new Cmca allotrope and the Cmmm and Pmmn allotropes share similar structural motifs, namely variations on arrangements of the same four-, six-, and eightfold ring patterns, which is the origin of their similar energetics. They also contain many sixfold diamondlike rings which may partially account for their relatively low energy and superhardness in carbon. Possibly, other energetically close structures could be conceived exhibiting variations of these motifs. This suggests that cold compressed CNT bundles could adopt a mix of different but structurally and energetically very close forms, as described by the family $\mathrm{Cmmm} / \mathrm{Pmmn} / \mathrm{Cmca}$.

Our search also produced the primitive tetragonal structure with 12 atoms per cell (T12) and space group $\mathrm{P}_{2} / \mathrm{ncm}$ that has been recently reported by Zhao et $a l .{ }^{35}$ In the present work we provide a detailed description of this structure and its relation to diamond (see Sec. III C 1 ), interpreting $\mathrm{P} 4_{2} / \mathrm{ncm}$ as a network of diamondlike tetrahedra made up of slabs of almost regular cornersharing tetrahedra alternatively rotated left and right, so that fivefold connectivity appears within each slab and adjacent slabs are bonded together by non-shared corners. We believe that this remarkable structure is the simplest that can be built by the stacking of slabs of tilted tetrahedra while preserving to a large degree a highly regular fourfold coordination for the sites. The $\mathrm{P} 4_{2} /$ ncm structure has been proposed ${ }^{35}$ as a candidate for both the Si-XIII phase (which is observed to coexist along with the Si-cd, Si-hd, Si-r8, and Si-bc8 phases in nanoindentation experiments ${ }^{6,7,20-22}$ ) and for an experimentally synthesized metastable Ge phase, ${ }^{70}$ and for this reason we compare our data for other candidate phases with those of $\mathrm{P} 4_{2} / \mathrm{ncm}^{71}$

We have also obtained two interesting and novel lowenergy structures, Pbam (with 24 atoms per cell) and $\mathrm{P} 4_{1} 2_{1} 2$ (20 atoms per cell), with orthorhombic and tetragonal symmetry, respectively, which are energetically competitive polymorphs and whose detailed de- scription will be given below. The orthorhombic Pbam structure and the tetragonal $\mathrm{P} 4_{1} 2_{1} 2$ structure are slightly expanded in volume with respect to the corresponding diamond structures, with zero-pressure equilibrium volumes about 2-3 \% larger than those of the corresponding cd structures, see Tables II-IV. The density of the $\mathrm{P} 6_{5} 22$ chiral framework structure reported previously ${ }^{42}$ is intermediate between those of Pbam/ $\mathrm{P} 4_{1} 2_{1} 2 / \mathrm{P} 4_{2} / \mathrm{ncm}$ and the type-I and II clathrates. The calculated phonon dispersion curves of these phases show that they are dynamically stable.

Note that the present Pbam structure with 24 atoms per cell is not the same as the Pbam structure with 16 atoms per cell proposed by Niu et al. for $R$ carbon, ${ }^{31}$ along with other orthorhombic structures. It is in particular significantly lower in energy. The present Pbam structure is energetically highly favorable in $\mathrm{C}, \mathrm{Si}$, and Ge. For carbon, Pbam is essentially degenerate in energy with the most favourable of the low-symmetry defect structures proposed recently by Botti et al., with monoclinic symmetry and 20, 22, and 24 atoms per cell, ${ }^{72}$ whereas in our study for silicon and germanium we have considered ex professo these hypothetical carbon structures and have found them to lie within $4 \mathrm{meV}$ of Pbam, which is close to the accuracy of the calculations. ${ }^{73}$ Apart from these structures, among those considered here, only the diamond forms and the type-II clathrate of germanium (Ge-clat34) are significantly lower in enthalpy at zero pressure than the corresponding Pbam structures, with the type-I germanium clathrate (Ge-clat46) having about the same zero-pressure entahlpy as Ge-Pbam (and a rather smaller density).

Low density clathrate structures of types I and II (here labelled clat46 and clat34, respectively) have been synthesized in $\mathrm{Si}, \mathrm{Ge}$, and $\mathrm{Sn},{ }^{16-18}$ although not in C. Group 14 clathrates have normally been synthesized by incorporating guest atoms, although a guestfree Ge clathrate has been formed. ${ }^{15}$ Our calculated results for the clathrates are in good agreement with earlier work. ${ }^{74-78}$ The high-density polymorphs bc $8, \mathrm{r} 8$ and st12 of $\mathrm{Si}$ and Ge are all considerably less stable than Pbam at zero pressure.

The $\mathrm{P} 4_{1} 2_{1} 2$ form is a chiral framework structure containing fivefold spirals of atoms, that is also energetically competitive, particularly in $\mathrm{Si}$, for which among the new polymorphs only the Pbam structure is lower in energy. In C, its enthalpy is very close to those of the $\mathrm{Cmmm} / \mathrm{Cmca}$ phases already mentioned in connection with cold compressed nanotubes. The $\mathrm{P} 4_{2} / \mathrm{ncm}$ structure is less stable than $\mathrm{P} 4_{1} 2_{1} 2$ in $\mathrm{Si}$, but more stable than $\mathrm{P} 4{ }_{1} 2{ }_{1} 2$ in $\mathrm{C}$ whereas for Ge both structures have approximately the same enthalpy. The two pure clathrate structures considered are also less stable than $\mathrm{P} 4_{1} 2_{1} 2$ in Si.

As has been observed previously, the calculated energy differences within the PBE are systematically larger than those from the PBEsol functional, and these are larger in turn than those from the LDA. One effect of this is 
that the coexistence pressures with the diamond phases for the high pressure phases (such as $\beta$-Sn) or the highdensity tetrahedral metastable phases (such as bc8) are larger with PBE than PBEsol or the LDA. The differences in energy between the PBE and the LDA or PBEsol are, however, quite small for the low-density tetrahedral polymorphs with equilibrium volumes close to the diamond phase such as Pbam, $\mathrm{P} 4_{2} / \mathrm{ncm}$, or $\mathrm{P} 4_{1} 2_{1} 2$, whereas for the high-density tetrahedral polymorphs such as bc8, r8, or st12 there is a significant difference between the results obtained with different exchange-correlation approximations, see Tables II-IV and Supplemental Material. This reflects the similarity in bonding in diamond and in phases such as Pbam, $\mathrm{P} 4_{2} / \mathrm{ncm}$, or $\mathrm{P} 4_{1} 2_{1} 2$. The bulk moduli of these and the other low-density structures obtained in the searches tend to be slightly below the bulk moduli of the corresponding diamond forms. In the case of carbon, for example, these are in the range $\sim 400-415 \mathrm{GPa}$ (cf. the calculated value for C-cd of 435 GPa).

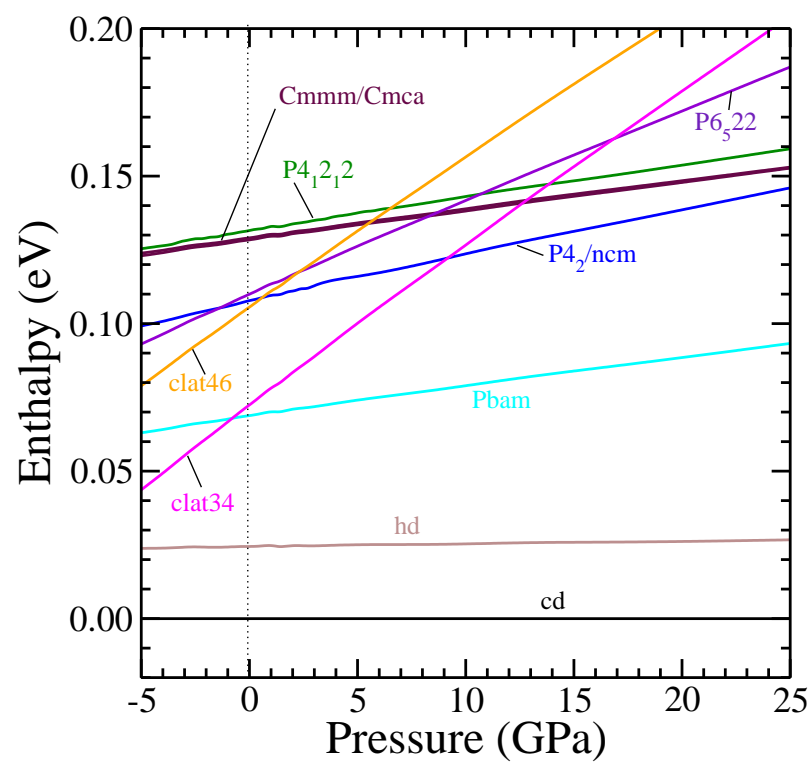

FIG. 1. (Color online) Enthalpy-pressure relations for various structural phases of carbon calculated using the PBE. The enthalpies are given with respect to the zero-pressure C-cd phase.

\section{Description of selected low-energy structures}

The three phases on which we focus next, $\mathrm{P} 4_{2} / \mathrm{ncm}$, Pbam, and $\mathrm{P} 4_{1} 2_{1} 2$, are structurally very different among themselves and with respect to the diamond structure. Topologically, all the atoms in these structures have a tetrahedral coordination of nearest neighbours, in some cases considerably distorted from a regular coordination, presenting five-, six-, and/or sevenfold rings of atoms. Each of these novel structures has similar characteristics

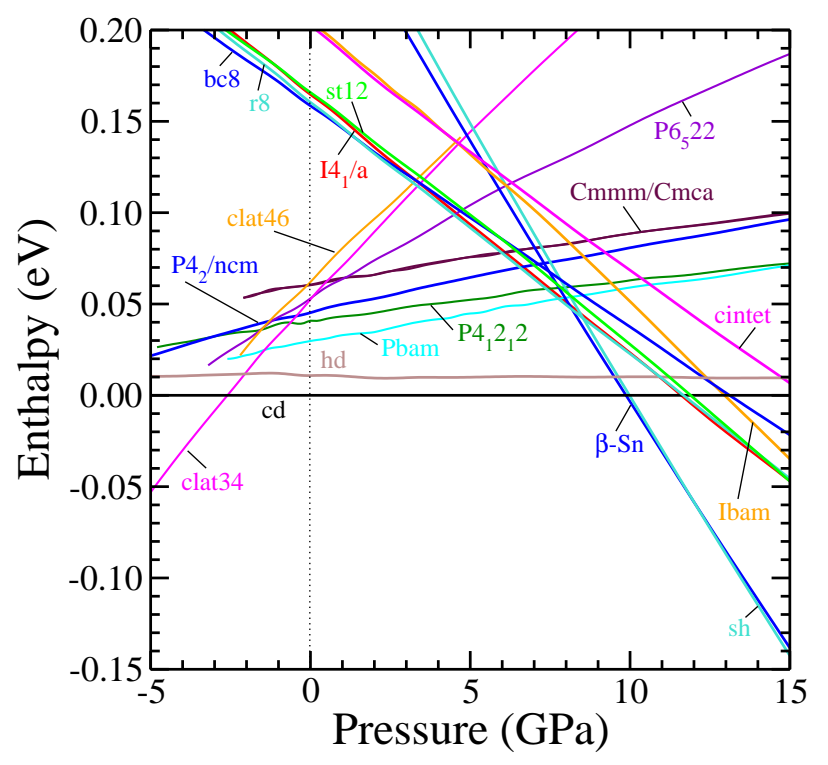

FIG. 2. (Color online) Enthalpy-pressure relations for various structural phases of silicon calculated using the PBE. The enthalpies are given with respect to the zero-pressure Si-cd phase.

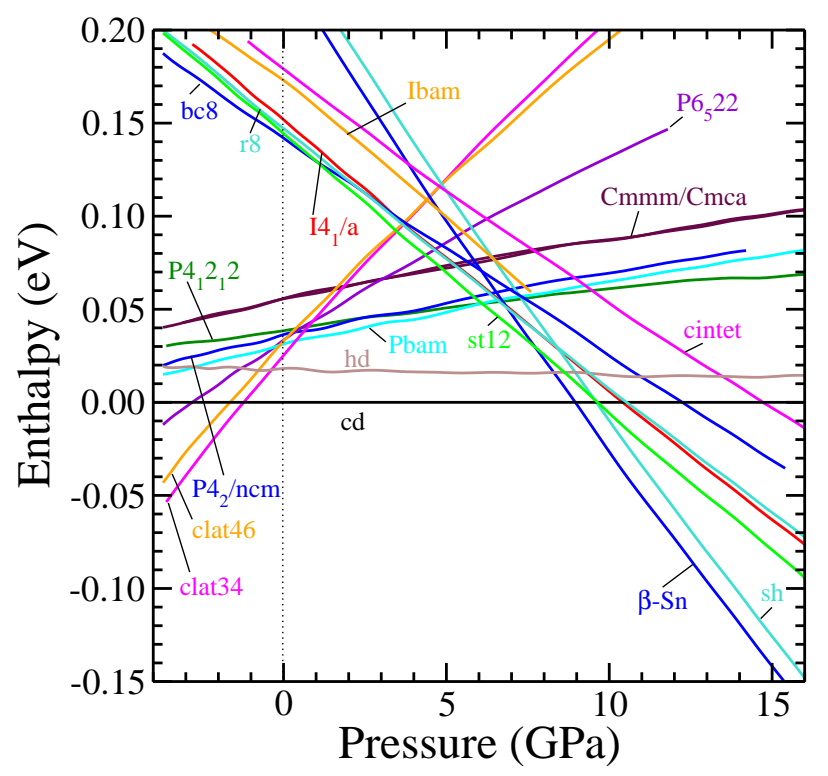

FIG. 3. (Color online) Enthalpy-pressure relations for various structural phases of germanium calculated using the PBE. The enthalpies are given with respect to the zero-pressure Ge-cd phase.

in all four materials studied. Hereafter we will discuss them in detail providing, for the sake of illustration, calculated numerical data (values of distances, angles etc) which correspond to $\mathrm{Si}$ at zero pressure, unless otherwise stated. In Table I we summarize the relevant crystallographic structural data for the $\mathrm{P} 4_{2} / \mathrm{ncm}, \mathrm{Pbam}$, and $\mathrm{P} 4_{1} 2{ }_{1} 2$ structures of $\mathrm{Si}$, at zero pressure, calculated using the PBE functional. Diffraction patterns of the struc- 
TABLE I. Crystallographic data for the I4 $1 / \mathrm{a}$, Cmca, $\mathrm{P} 4_{2} / \mathrm{ncm}$, Pbam, and $\mathrm{P} 4_{1} 2_{1} 2$ structures of $\mathrm{Si}$ at zero pressure, calculated using the PBE: space group (SG), lattice parameters, and atomic sites (Wyckoff positions). Those internal coordinates fixed by symmetry are given as integer fractions, to aid recognition.

\begin{tabular}{ll}
\hline \hline $\mathrm{I} 4_{1} / \mathrm{a}$ & $\mathrm{SG} I 4_{1} / a$, No.88 \\
& $\begin{array}{l}a=6.676 \AA, c=6.514 \AA \\
\text { Sil } \quad 16 \mathrm{f} \quad(-0.0962,-0.0994,0.1170)\end{array}$
\end{tabular}

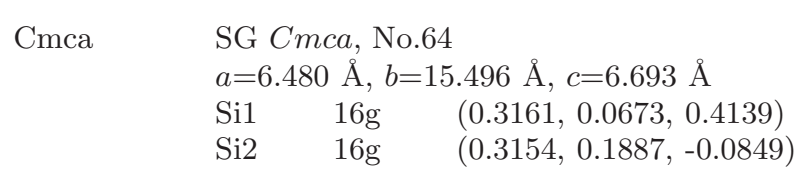

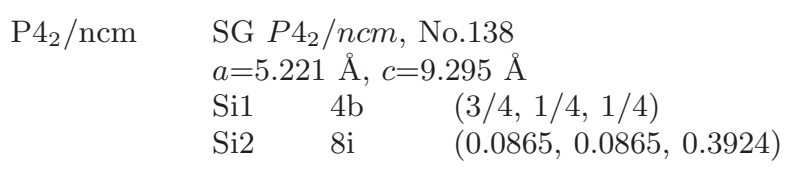

\begin{tabular}{|c|c|c|c|}
\hline \multirow[t]{7}{*}{ Pbam } & \multicolumn{3}{|c|}{$\begin{array}{l}\text { SG Pbam, No. } 55 \\
a=11.774 \AA, b=11.081 \AA, c=3.863 \AA\end{array}$} \\
\hline & Si1 & $4 \mathrm{~h}$ & $(0.6158,0.2400,1 / 2)$ \\
\hline & $\mathrm{Si} 2$ & $4 \mathrm{~h}$ & $(0.4625,0.1005,1 / 2)$ \\
\hline & $\mathrm{Si} 3$ & $4 \mathrm{~h}$ & $(0.9081,0.9569,1 / 2)$ \\
\hline & $\mathrm{Si} 4$ & $4 \mathrm{~g}$ & $(0.7705,0.7075,0)$ \\
\hline & $\mathrm{Si} 5$ & $4 \mathrm{~g}$ & $(0.8040,0.0124,0)$ \\
\hline & Si6 & $4 \mathrm{~g}$ & $(0.1501,0.6240,0)$ \\
\hline
\end{tabular}

\begin{tabular}{|c|c|c|}
\hline \multirow[t]{4}{*}{$\mathrm{P} 4_{1} 2_{1} 2$} & \multicolumn{2}{|c|}{$\begin{array}{l}\text { SG } P 4_{1} 2_{1} 2, \text { No. } 92 \\
a=8.903 \AA, c=5.261 \AA\end{array}$} \\
\hline & Si1 $8 b$ & $(0.4312,0.1390,0.7001)$ \\
\hline & $\mathrm{Si} 2$ & $(0.4752,0.6504,0.4205)$ \\
\hline & $\mathrm{Si} 3$ & $(0.2011,0.2011,1 / 2)$ \\
\hline
\end{tabular}

tures simulated using the FULLPROF software ${ }^{79}$ are shown in Fig. 4. Further details of these structures are given in the Supplemental Material. ${ }^{36}$

\section{The P42/ncm structure as a network of tilted tetrahedra}

$\mathrm{P} 4_{2} / \mathrm{ncm}$ is a remarkably simple structure that can be understood as a stacking of (001) slabs of diamondlike corner-sharing tetrahedra, with adjacent tetrahedra within each slab alternately tilted clockwise and anticlockwise by approximately $20^{\circ}$, see Fig. 5. The centers of the tetrahedra correspond to the Sil sites of the $\mathrm{P} 4_{2} /$ ncm structure (red sites in Fig. 5) and their corners correspond to the $\mathrm{Si} 2$ sites (blue). There are only three free internal parameters in $\mathrm{P} 4_{2} / \mathrm{ncm}$, all them related to the Si2 corner sites, whereas the Si1 center sites form a simple tetragonal lattice. Within each of these tetrahe-

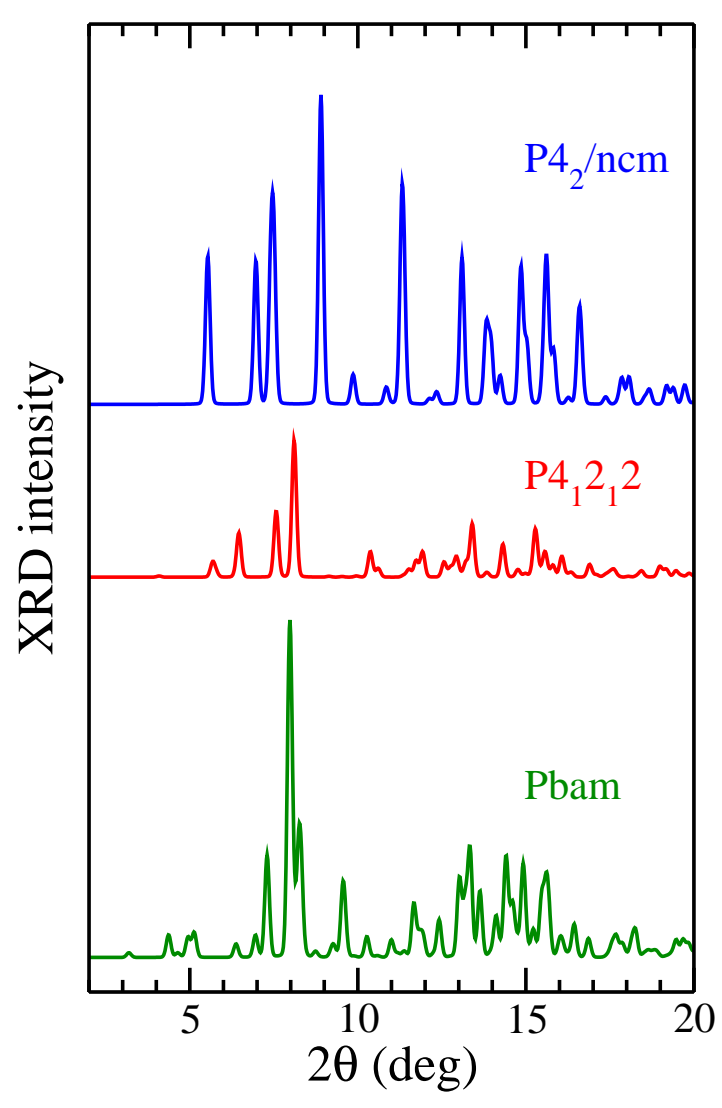

FIG. 4. (Color online) Simulated powder XRD patterns of the $\mathrm{P} 4_{2} / \mathrm{ncm}, \mathrm{P} 4_{1} 2_{1} 2$, and Pbam phases of $\mathrm{Si}$ at zero pressure, calculated within the PBE approximation. The x-ray wavelength used was $0.44864 \AA$.

dral slabs, the rotation of adjacent tetrahedra in opposite sense brings their corners together, with the formation of new bonds between them (blue rods in Fig. 5b), above and below the plane of the centers and orientated along $\langle 110\rangle$ directions. When two of these tetrahedral slabs, oppositely orientated (Fig. 5b and c), are stacked along the $z$ axis, new inter-slab bonds are established between the corners of each tetrahedron and the corners of the opposite tetrahedra immediately above and below it (green rods in Fig. 5d). Note that the centers of the tetrahedra in different slabs are strictly aligned along the stacking direction, whereas the orientation of the stacked tetrahedra are alternately tilted clockwise and anti-clockwise. As a result of intra- and inter-slab bonding of tetrahedral corners, each Si2 corner site is linked to two other $\mathrm{Si} 2$ corner sites (as well as to two Si1 center sites), with the formation of staggered chains of Si2 corner sites running along $\langle 110\rangle$ directions.

It is quite remarkable that while the tilting and rebonding reduces the symmetry of the $\mathrm{P} 4_{2} / \mathrm{ncm}$ tetrahedral arrangement with respect diamond, so that in $\mathrm{P} 4_{2} / \mathrm{ncm}$ the tetrahedral units become tetragonal disphenoids, these units are actually very close to regular tetrahedra. The Si1 center sites have an almost ideal tetrahedral Si2 en- 
vironment, with all four bonds of equal length and nearideal tetrahedral angles. The tetrahedral units perform largely as rigid units in the $\mathrm{P} 4_{2} / \mathrm{ncm}$ structure.

The tilting and subsequent intra-slab corner-bonding leads to the appearance of fivefold rings made up of two Si1 tetrahedra centers and three Si2 corners, see Fig. 5b. Each of these fivefold rings is a slightly buckled and symmetrical pentagon with four out of its five sides having the same length, and with three different angles, which deviate only moderately from the angle of $108^{\circ}$ of a regular pentagon. The stacking of opposite tetrahedral slabs and accompanying corner-bonding further results in twisted sixfold rings $(2 \times \mathrm{Si} 1,4 \times \mathrm{Si} 2)$ with alternating orientation along the $z$ axis, noted by Zhao et al. ${ }^{35}$ and of sevenfold rings $(2 \times \mathrm{Si} 1,5 \times \mathrm{Si} 2)$ in a chair-like configuration, also alternating in orientation along the stacking direction. The twisted sixfold rings are quite unlike those in the diamond structure. There is only one type of five-, six-, and sevenfold ring.

There are only three different bond lengths in P $4_{2} / \mathrm{ncm}$ : one $\left(d_{1}\right)$ between Si1 and Si2 sites (that is, from the center to each corner of the quasi-regular tetrahedra), another between neighboring Si2 sites in the same slab $\left(d_{2}\right)$, and a third between Si2 sites in adjacent slabs $\left(d_{3}\right)$. These three different bond lengths are represented, respectively, by red, blue, and green rods in Fig. 5. The tetrahedral distance $d_{1}$ is very similar to that of the diamond phase $(2.37 \AA)$ and shows a similar compressibility under applied pressure, which is in line with the fact that the tetrahedral units of $\mathrm{P} 4_{2} / \mathrm{ncm}$ remain very much diamond-like in nature. The inter-slab distance $d_{3}$ is also similar though it has a slightly larger compressibility, while the intra-slab distance $d_{2}$ is larger than both $d_{1}$ and $d_{3}$ and it also shows a larger compressibility. There are also six different bond angles, ${ }^{81}$ with only one angle deviating significantly $\left(122.5^{\circ}\right)$ from the ideal tetrahedral value $\left(109.47^{\circ}\right)$. This large angle corresponds to the chains of bonds between Si2 tetrahedra corners running along $\langle 110\rangle$ directions. The bond angles in $\mathrm{P} 4_{2} / \mathrm{ncm}$ vary very little when the structure is compressed. The large Si2-Si2-Si2 angle increases by about $1.5^{\circ}$ in the range from $-5 \mathrm{GPa}$ to $10 \mathrm{GPa}$, while the rest of the angles change by tenths of a degree. The tilting of the tetrahedra is locked by intra- and inter-slab bonding and the aspect of the $\mathrm{P} 4_{2} /$ ncm structure would not change much even over a substantial pressure range.

\section{The Pbam structure}

With six inequivalent sites and twelve free internal parameters, Pbam has a larger variation of tetrahedral environments than $\mathrm{P} 4_{2} / \mathrm{ncm}$, and yet many elements of Pbam remain remarkably similar to those of diamond with which a useful comparison can be established. In Fig. 6 we represent the Pbam structure in a way that allows comparison to the diamond structure, when the latter is viewed along its $\langle 110\rangle$ direction. In analogy to

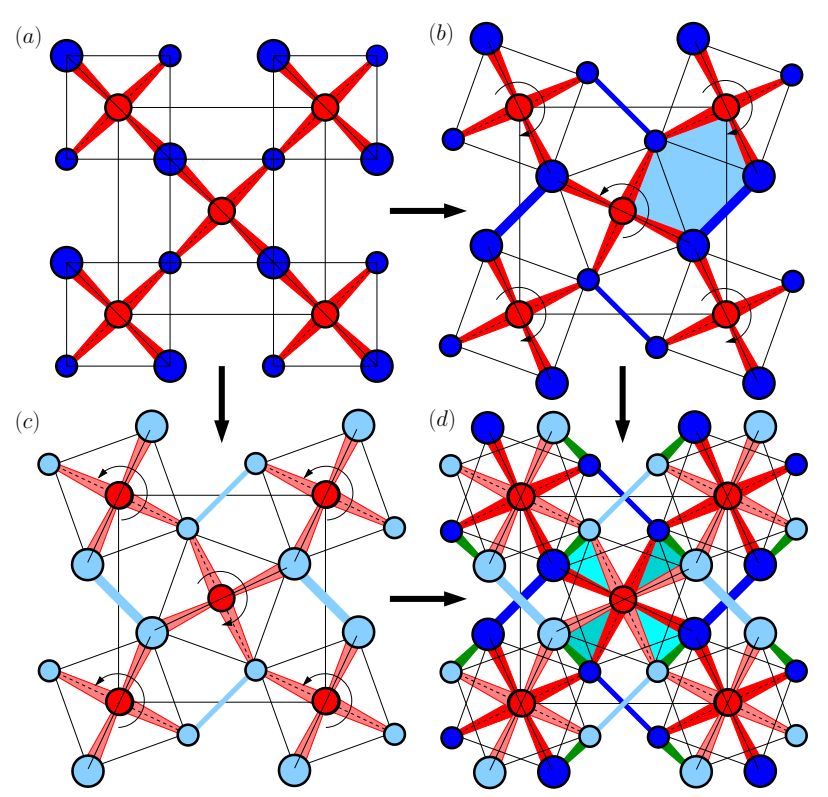

FIG. 5. Construction of $\mathrm{P} 4_{2} /$ ncm as a network of tilted diamond-like tetrahedra. Panel (a) shows a single slab of corner-sharing regular tetrahedra of the diamond structure viewed along the (001) direction. The projected tetrahedra and conventional cubic cell are shown in black. The centers of the tetrahedra are shown in red and their corners in blue, with the different sizes of the atoms and varying thickness of the bonds used to give a sense of perspective. Panels (b) and (c) show two adjacent slabs (lower and upper, respectively) of tilted tetrahedra that alternate stacked along the $z$ axis to give the $\mathrm{P} 4_{2} / \mathrm{ncm}$ structure, as shown in (d). ${ }^{80}$ Note the opposite orientation of the tetrahedral bonds and the tilting of the tetrahedra in the two adjacent slabs. Intra-slab bonds (parallel to the plane of projection), resulting from the tilting of the tetrahedral units within each slab, are represented by blue rods, while inter-slab bonds, arising from the stacking of the slabs, are represented by green rods. Intra-slab bonding leads to characteristic fivefold rings, one of which is depicted in blue in (b), while inter-slab bonding leads to buckled sixfold rings, two of which are shown in profile in $(d)$.

diamond, we see stacks of zigzag chains in Pbam, two per cell, linking the three 4g-type sites Si4-6 (red sites in Fig. 6 ). These distorted diamond-like chains are contained in (001) projection planes and run along the $\langle 010\rangle$ direction. The three 4h-type sites Si1-3 (blue sites) form in turn two staggered planar chains along the same direction but at different (001) planes from the $4 \mathrm{~g}$-chains and intercalated between them. Also note the relative displacements and different orientations of consecutive $4 \mathrm{~g}$ and $4 \mathrm{~h}$ chains. Adjacent $4 \mathrm{~g}$ and $4 \mathrm{~h}$ chains are connected by three different types of bond which form zigzag diamond-like chains running perpendicular to the projection plane, each linking one type of $4 \mathrm{~g}$ site to one type of $4 \mathrm{~h}$ site (Si1-Si6, Si2$\mathrm{Si} 4$, and $\mathrm{Si} 3-\mathrm{Si} 5$ ), so each $4 \mathrm{~g}$ site (respectively, $4 \mathrm{~h}$ site) is linked to two $4 \mathrm{~g}(4 \mathrm{~h})$ sites on the same (001) plane and to two $4 \mathrm{~h}(4 \mathrm{~g})$ sites of the same type, above and below the plane. The orientations of these three different 
connecting $4 \mathrm{~g}-4 \mathrm{~h}$ chains of bonds deviate greatly from the regular orientation found in the diamond structure (which would correspond to a (010) plane in Pbam) but the chains themselves are very little distorted compared with equivalent chains in diamond.

The main topological difference between diamond and Pbam arises from the intra-chain connectivity within the $4 \mathrm{~h}$ slabs in which drastic changes lead to the formation of the fivefold and sevenfold rings shown in Fig. 6. There are in fact no other major topological changes either within the $4 \mathrm{~g}$ slabs or within the interconnecting $4 \mathrm{~g}-4 \mathrm{~h}$ chains. A simple interpretation of Pbam is that it consists in a certain pattern of shearing of atomic slabs in diamond such that breaking and formation of intra-slab bonds occurs only within alternate slabs (the stacks of $4 \mathrm{~h}$ chains in Pbam) whereas the connecting chains of interslab bonds are not affected beyond a small deformation of bond lengths and angles. All in all, two bonds are broken and reformed per $4 \mathrm{~h}$ chain and cell, which drastically transforms the $4 \mathrm{~h}$ intra-slab connectivity giving rise to fivefold and sevenfold rings perpendicular to the shearing direction, though some sixfold diamond-like rings are also preserved, see Fig. 6. Whereas the 4 h slabs undergo an important reconstruction, the topology of the $4 \mathrm{~g}$ slabs remains essentially unchanged (though deformed) and the $4 \mathrm{~g}-4 \mathrm{~h}$ chains of connecting bonds are mostly unaltered (though rotated from their initial orientation by the intra- $4 \mathrm{~h}$ rebonding). These changes are accompanied by a contraction in the $y$ direction and elongation in the $x$ direction with respect to diamond.

There is one type each of fivefold, sixfold, and sevenfold rings in the $x y$ projection of Pbam shown in Fig. 6, which appear in four different orientations. ${ }^{82}$ Apart from the sixfold rings shown in Fig. 6, the Pbam structure preserves a large number of other sixfold chair-like rings of the diamond structure perpendicular to the $x y$ plane which are shown in profile in this figure. (There are also eightfold rings perpendicular to the $x y$ plane formed by the rebonding of the 4 h slabs).

Due to the large number of free parameters of the Pbam structure, there are ten different nearest neighbor distances and twenty one different bond angles. Distances vary between 2.33 and $2.42 \AA$ (cf. the calculated value for the diamond structure of $2.37 \AA$ ) with most distances within $0.4 \%$ of the diamond value and a mean distance of $2.37 \AA$. Bonds within the $4 \mathrm{~g}$ slabs are somewhat shorter than diamond bonds whereas those within the $4 \mathrm{~h}$ slabs are somewhat longer, with the longest bond occurring between the $\mathrm{Si} 2$ and $\mathrm{Si} 3$ sites that form the pentagonal rings. Bond compressibility is also similar to that in diamond, although the two Si1-Si2 and Si3-Si3 bonds within the $4 \mathrm{~h}$ slabs have somewhat larger compressibility and one Si5-Si6 bond within the $4 \mathrm{~g}$ slabs has a smaller compressibility.

There is also a considerable angular distortion with respect to the ideal tetrahedral angle. Not surprisingly, the largest angle $\left(123.9^{\circ}\right)$ occurs within the heptagonal rings (two other different angles in the sevenfold rings

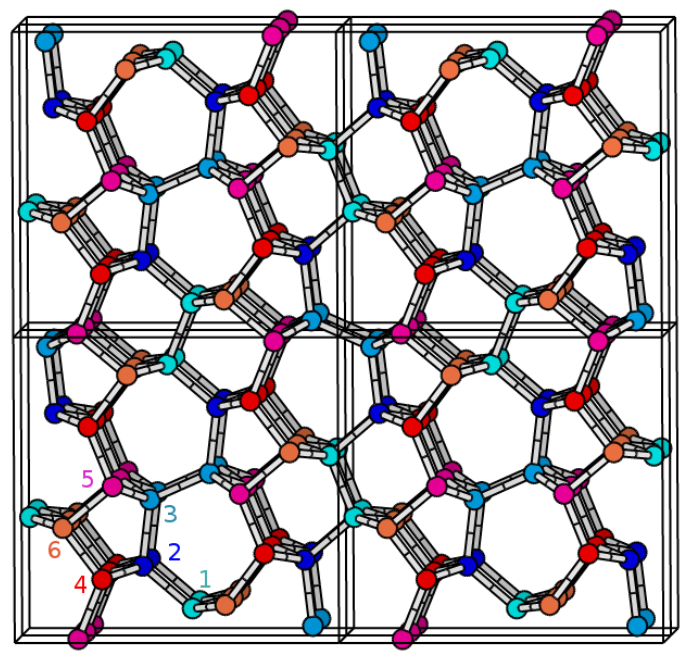

FIG. 6. (Color online) A slab of the Pbam structure viewed along the $z$ axis, with unit cells shown in black and a modicum of perspective so as to reveal the connectivity among sites. The six inequivalent sites are labelled and shown in different colors: the 4g-type sites are depicted in three different hues of red, while blue is used for the three 4h-type sites. The fivefold and sevenfold rings of the structure (as well as sixfold diamond-like rings) are all visible in this projection. Note that the red $4 \mathrm{~g}$ sites and the blue $4 \mathrm{~h}$ sites are each in different projection planes a distance $c / 2$ apart.

are close by, at $120.6^{\circ}$; cf. the angle of a regular heptagon of $\left.128.57^{\circ}\right)$. The minimum angle $\left(92.6^{\circ}\right)$ occurs at the vertex of the fivefold rings (where the rest of the angles vary between $101.4^{\circ}$ and $102.7^{\circ}$ ). In spite of these distortions it is quite remarkable that the Si1 sites at the junction of two adjacent sixfold rings have a tetrahedral environment of neighbors very similar to that of diamond sites. In fact, the sixfold rings themselves are very similar to those present in ideal diamond, with angles deviating slightly from the ideal tetrahedral angle (from $107.7^{\circ}$ to $110.6^{\circ}$ ) and a mean angle of $109.3^{\circ}$. The angular deviations with respect to diamond are also minimal along the three different zigzag chains running perpendicular to the $x y$ plane $\left(108.6^{\circ}, 109.2^{\circ}\right.$, and $\left.110.1^{\circ}\right)$ where the bond lengths are also very similar to diamond. As in $\mathrm{P} 4_{2} / \mathrm{ncm}$, the bond angles remain very stable under compression with a maximum variation of $1.8^{\circ}$ from $-5 \mathrm{GPa}$ to $10 \mathrm{GPa}$, but in fact most angles vary only by tenths of a degree.

\section{The P41212 structure: A new chiral framework with tetragonal symmetry}

Whereas Pbam and $\mathrm{P} 4_{2} / \mathrm{ncm}$ share some common elements with the diamond structure, $\mathrm{P} 4_{1} 2_{1} 2$ does not show any obvious similarity with diamond, beyond being also a tetrahedral network. With three different types of site, 
six different bond lengths and sixteen different bond angles, such a network is quite unlike the regular tetrahedral network found in diamond. Instead, $\mathrm{P} 4_{1} 2_{1} 2$ has an obvious similarity to the chiral framework structure (CFS) of $P 6_{5} 22$ symmetry previously discovered by Pickard and Needs. ${ }^{42,83}$

In Fig. 7 we show the $\mathrm{P} 4_{1} 2_{1} 2$ structure viewed from the direction of its main rotation axis (cf. the Supplemental Material ${ }^{36}$ and Fig. 1 of Ref. 42 for a similar plot of the CFS-P6 622 structure). The two-dimensional pattern of this projection corresponds to the so called Cairo pentagonal tiling, which is reported to appear in Islamic decoration. ${ }^{84}$ Like the CFS-P 6522 structure, the new $\mathrm{P} 4{ }_{1} 2_{1} 2$ structure can be viewed as made of interconnected spiral chains of bonds running both clockwise and anti-clockwise along the $z$ axis, with some portions of open space without clearly defined cavities (as, for example, in the known clathrate structures). In $\mathrm{P} 4_{1} 2_{1} 2$ there is one type of threefold spiral, three types of fourfold spirals and one type of fivefold spiral (whereas in CFSP 6522 there is one type each of threefold, fourfold and sixfold spirals). The presence of fivefold spirals (Si1-Si2Si2-Si1-Si3) in $\mathrm{P} 4_{1} 2_{1} 2$ is a rather unusual feature, which is unique and characteristic of this structure among others of group-14 elements previously reported or that we have found in our searches. There is also a large proportion of fivefold rings (of which there are two types) as well as sixfold rings. Angular distortions from the ideal tetrahedral angle are considerable, with the maximum angle $\left(126.9^{\circ}\right)$ appearing along the fivefold spiral chains (Si1-Si3-Si1) and the minimum angle $\left(99.7^{\circ}\right)$ for Si1-Si2-Si2. Distances range from 2.35 to $2.39 \AA$ (cf. the value of $2.37 \AA$ for the diamond phase). The response of bonds to compression appears to be similar to diamond with just one bond having a somewhat larger compressibility. There is a large variation in the orientation of these bonds.

Both CFS-P6 522 and CFS-P $4_{1} 2_{1} 2$ (which we could rename CFS-6 and CFS-5, respectively, on account of the order of the main spirals, in each case) have rather similar energies, but the equilibrium volume of CFS-P $6_{5} 22$ is larger as it is based on sixfold instead of fivefold spirals, which results in a more open structure, with larger portions of empty space. Though they have not appeared during our structural searches, one may legitimately wonder whether other chiral structures of the same kind but based upon sevenfold or even higher order spirals would be energetically competitive and feasible as stable phases.

\section{Band structures}

The band structures and electronic densities of states (EDoS) of the Pbam, $\mathrm{P} 4_{1} 2_{1} 2$, and $\mathrm{P} 4_{2} / \mathrm{ncm}$ structures of $\mathrm{Si}$ at zero pressure are shown in Fig. 8, and similar data for $\mathrm{C}$ and $\mathrm{Ge}$ are provided in the Supplemental Material. ${ }^{36}$ The calculated band gaps are expected to be underestimated, as is usual in DFT calculations using

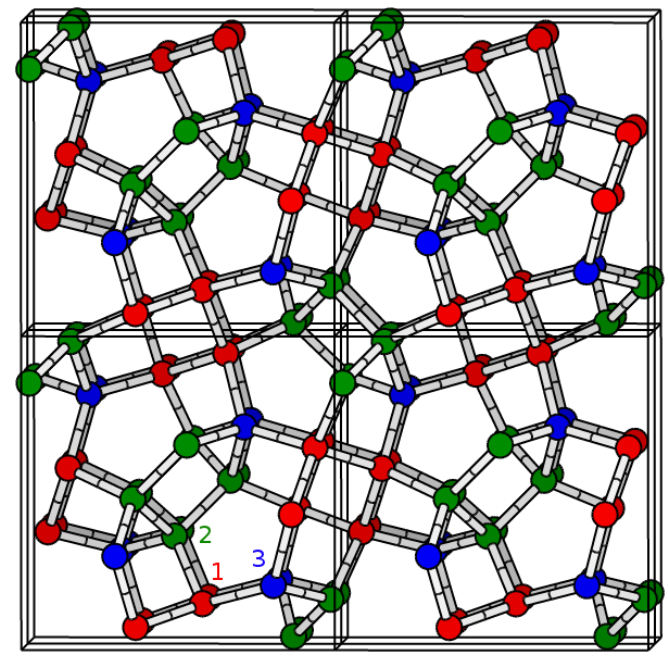

FIG. 7. (Color online) A slab of the $\mathrm{P} 4_{1} 2_{1} 2$ structure of $\mathrm{Si}$ at zero pressure, viewed along the direction of its fourfold rotation axis. A modicum of perspective has been introduced to facilitate visualization of the connectivity among sites. The three inequivalent sites are labelled and shown in different colours.

TABLE II. The equilibrium volume per atom, $V_{0}$, bulk modulus, $B_{0}$, pressure derivative of the bulk modulus, $B_{0}^{\prime}$, and the difference in energy from the corresponding diamond phase at zero pressure, $\Delta E$, for various structural phases of carbon. These calculated results were obtained with the VASP code and PBE density functional. (See also Supplemental Material for LDA and PBEsol results, as well as other phases.)

\begin{tabular}{lcccc}
\hline \hline Carbon & $V_{0}\left(\AA^{3}\right)$ & $B_{0}(\mathrm{GPa})$ & $B_{0}^{\prime}$ & $\Delta E(\mathrm{meV})$ \\
\hline cd & 5.708 & 435 & 3.51 & 0 \\
\hline hd & 5.722 & 433 & 3.60 & 25 \\
\hline Pbam & 5.873 & 416 & 3.60 & 69 \\
\hline $\mathrm{P} 4_{1} 2_{1} 2$ & 5.897 & 413 & 3.61 & 132 \\
\hline $\mathrm{P} 4_{2} / \mathrm{ncm}$ & 5.971 & 403 & 3.64 & 108 \\
\hline Cmca & 5.874 & 413 & 3.72 & 128 \\
\hline Cmmm & 5.870 & 413 & 3.67 & 129 \\
\hline $\mathrm{P} 6_{5} 22$ & 6.232 & 389 & 3.51 & 110 \\
\hline $\mathrm{I} 4_{1} / \mathrm{a}$ & 5.696 & 346 & 4.18 & 858 \\
\hline bc8 & 5.616 & 386 & 3.84 & 697 \\
\hline r8 & 5.658 & 363 & 3.98 & 819 \\
\hline st12 & 5.650 & 395 & 3.74 & 886 \\
\hline Ibam & 5.771 & 372 & 3.64 & 956 \\
\hline cintet & 5.498 & 428 & 3.72 & 1115 \\
\hline clat34 & 6.602 & 372 & 3.58 & 72 \\
\hline clat46 & 6.546 & 368 & 3.61 & 106 \\
\hline \hline
\end{tabular}

semi-local density functionals such as PBE, but the differences between the calculated band gaps are expected to be more accurate, by which useful estimations can be obtained.

The C-Pbam, C-P $4_{1} 2_{1} 2$, and $\mathrm{C}-\mathrm{P} 4_{2} / \mathrm{ncm}$ phases are insulators with minimum band gaps calculated to be 4.57 , 4.70 , and $3.74 \mathrm{eV}$, respectively (cf. the calculated band 
TABLE III. As Table II, but for silicon.

\begin{tabular}{lcccc}
\hline \hline Silicon & $V_{0}\left(\AA^{3}\right)$ & $B_{0}(\mathrm{GPa})$ & $B_{0}^{\prime}$ & $\Delta E(\mathrm{meV})$ \\
\hline cd & 20.45 & 88.9 & 4.1 & 0 \\
\hline hd & 20.43 & 88.6 & 4.4 & 11 \\
\hline Pbam & 20.98 & 85.0 & 4.2 & 29 \\
\hline $\mathrm{P} 4_{1} 2_{1} 2$ & 20.87 & 85.0 & 4.1 & 41 \\
\hline $\mathrm{P} 4_{2} / \mathrm{ncm}$ & 21.11 & 83.2 & 4.4 & 45 \\
\hline Cmca & 20.96 & 84.5 & 4.2 & 61 \\
\hline Cmmm & 20.95 & 84.8 & 4.2 & 61 \\
\hline $\mathrm{P} 6_{5} 22$ & 22.18 & 80.9 & 3.0 & 53 \\
\hline $\mathrm{I} 4_{1} / \mathrm{a}$ & 18.17 & 77.1 & 4.4 & 165 \\
\hline bc8 & 18.45 & 83.6 & 4.2 & 159 \\
\hline r8 & 18.22 & 78.8 & 4.3 & 160 \\
\hline st12 & 18.35 & 71.3 & 3.1 & 166 \\
\hline Ibam & 18.30 & 59.1 & 2.8 & 204 \\
\hline cintet & 18.12 & 96.0 & 4.4 & 203 \\
\hline clat34 & 23.56 & 76.1 & 4.3 & 52 \\
\hline clat46 & 23.27 & 75.8 & 4.2 & 67 \\
\hline \hline
\end{tabular}

TABLE IV. As Table II, but for germanium.

\begin{tabular}{lcccc}
\hline \hline Germanium & $V_{0}\left(\AA^{3}\right)$ & $B_{0}(\mathrm{GPa})$ & $B_{0}^{\prime}$ & $\Delta E(\mathrm{meV})$ \\
\hline cd & 24.18 & 59.2 & 4.6 & 0 \\
\hline hd & 24.13 & 59.5 & 4.6 & 17 \\
\hline Pbam & 24.83 & 56.2 & 4.8 & 31 \\
\hline $\mathrm{P} 4_{1} 2_{1} 2$ & 24.58 & 57.5 & 4.8 & 39 \\
\hline $\mathrm{P} 4_{2} / \mathrm{ncm}$ & 24.82 & 56.7 & 4.7 & 35 \\
\hline Cmca & 24.81 & 56.1 & 4.7 & 55 \\
\hline Cmmm & 24.81 & 56.3 & 4.6 & 54 \\
\hline $\mathrm{P} 6_{5} 22$ & 26.04 & 53.2 & 4.7 & 33 \\
\hline $\mathrm{I} 4_{1} / \mathrm{a}$ & 21.74 & 52.8 & 5.0 & 146 \\
\hline bc8 & 22.22 & 56.5 & 4.4 & 137 \\
\hline r8 & 21.83 & 53.6 & 4.9 & 142 \\
\hline st12 & 21.73 & 49.2 & 4.7 & 139 \\
\hline Ibam & 21.92 & 41.0 & 5.0 & 165 \\
\hline cintet & 21.94 & 61.7 & 4.7 & 173 \\
\hline clat34 & 27.50 & 51.9 & 4.8 & 25 \\
\hline clat46 & 27.10 & 51.5 & 4.8 & 32 \\
\hline \hline
\end{tabular}

gap of C-cd of $4.13 \mathrm{eV})$. The occupied bandwidths are $20.8 \mathrm{eV}$ (C-Pbam), $19.7 \mathrm{eV}\left(\mathrm{C}-\mathrm{P} 4_{1} 2_{1} 2\right)$, and $19.9 \mathrm{eV}$ (C$\left.\mathrm{P} 4_{2} / \mathrm{ncm}\right)$, which are somewhat smaller than the calculated bandwidth of the diamond phase, $21.4 \mathrm{eV}$ (cf. the experimental value of of the bandwidth of C-cd, $23.0 \pm 0.2$ eV, Ref. ${ }^{85}$ ).

The corresponding minimum band gaps for the $\mathrm{Si}$ structures are $0.86 \mathrm{eV}$ (Si-Pbam), $1.23 \mathrm{eV}$ (Si-P4 $4_{1} 2_{1} 2$ ), and $0.80 \mathrm{eV}\left(\mathrm{Si}-\mathrm{P} 4_{2} / \mathrm{ncm}\right)$, which are larger than the gap of $0.63 \mathrm{eV}$ calculated for the diamond structure using PBE-DFT, which is in turn approximately half the experimental value of $1.17 \mathrm{eV}$. If we add to the band gaps of $\mathrm{Si}-\mathrm{Pbam}, \mathrm{Si}-\mathrm{P} 4_{1} 2_{1} 2$, and $\mathrm{Si}-\mathrm{P} 4_{2} / \mathrm{ncm}$ a correction equal to the difference between the theoretical and experimental band gaps of Si-cd we obtain band gaps of about $1.4 \mathrm{eV}$ (Si-Pbam), $1.77 \mathrm{eV}\left(\mathrm{Si}-\mathrm{P} 4_{1} 2_{1} 2\right)$, and $1.34 \mathrm{eV}$ (Si$\mathrm{P} 4_{2} / \mathrm{ncm}$ ). These gaps are larger than in Si-cd, and are considerably closer to the values around $1.5 \mathrm{eV}$ that are
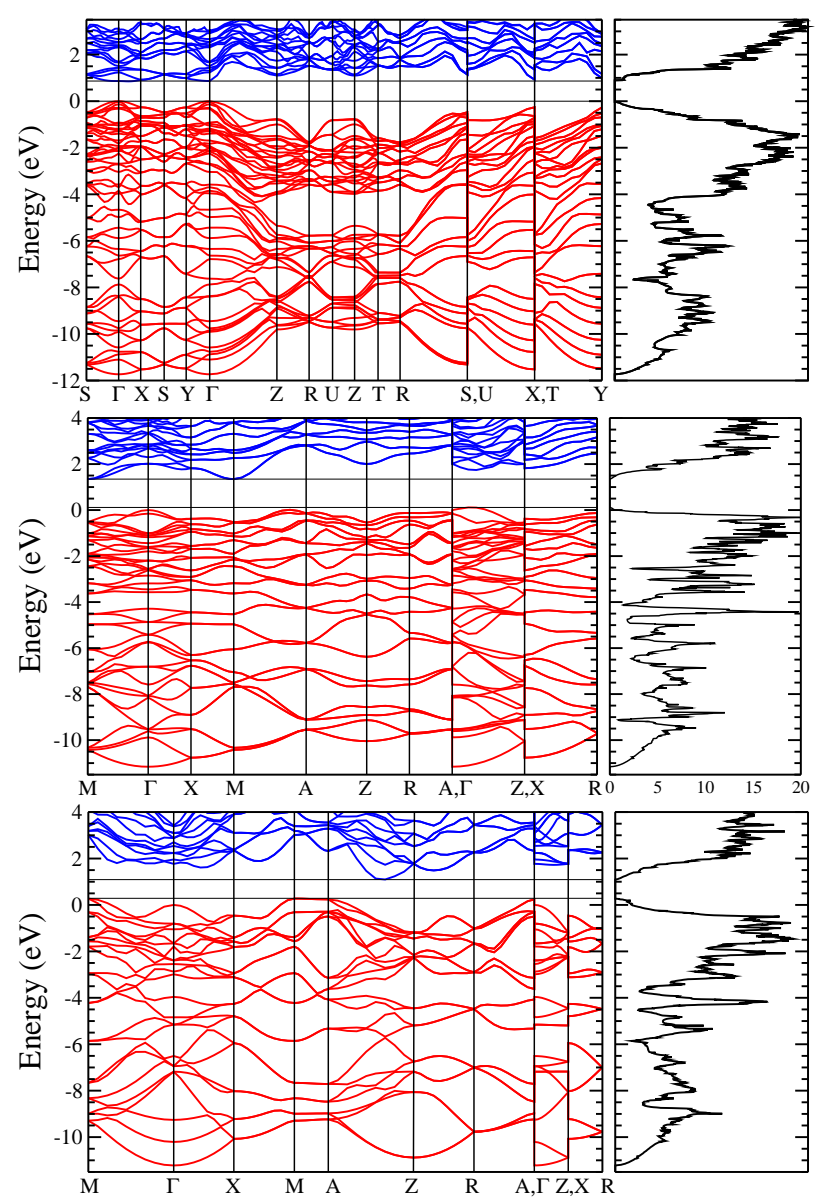

FIG. 8. (Color online) Electronic band structures and densities of states of the Pbam, $\mathrm{P} 4_{1} 2_{1} 2$, and $\mathrm{P} 4_{2} / \mathrm{ncm}$ structures of $\mathrm{Si}$ at zero pressure. The valence bands are shown in red and the conduction bands in blue, and the band gap regions are indicated by parallel black lines. The zeros of energy are at the top of the valence band at the zone center $\Gamma$ point. The panel on the right of each plot shows the total density of states.

optimal for photovoltaic applications.

In particular, the top of the valence band of Si-Pbam is located at the zone center $\Gamma$, where the (corrected) direct band gap is $\sim 1.4 \mathrm{eV}$, and therefore this phase could be of technological interest. (The bottom of the conduction band has a rather flat variation around $\Gamma$, along the $\Gamma$ $X$ and $\Gamma-Y$ directions, with an extremely tiny dip of the order of $0.01 \mathrm{eV}$, and for this reason one could also regard the gap as quasi direct. Due to the small axial ratios $c / a$ and $b / a$ of the Pbam cell, both the $X$ and $Y$ points are close to the zone center in Si-Pbam, see Fig. 8.) The very small dispersion of the conduction bands around $\Gamma$ suggests that the joint density of states for electron excitation is large. In contrast, the band structures of both $\mathrm{Si}-\mathrm{P} 4_{1} 2_{1} 2$ and $\mathrm{Si}-\mathrm{P} 4_{2} / \mathrm{ncm}$ show distinct indirect band gaps, as Si-cd.

The Ge-Pbam phase has a calculated direct band gap at the zone center close to zero, while Ge-P $4_{1} 2_{1} 2$ and 
Ge-P $4_{2} / \mathrm{ncm}$ have calculated indirect gaps of about 1.00 $\mathrm{eV}$ and $0.30 \mathrm{eV}$, respectively. Analogous calculations for Ge-cd give a band gap of approximately zero (cf. the experimental value of $0.67 \mathrm{eV}$ ), thus the corrected gaps for the tetrahedral polymorphs are also expected to be larger than for the Ge-cd phase, as found in Si.

A common feature of the band structures of the lowdensity polymorphs of group 14 phases is a weakening of the $s p$ hybridization arising from the substantial deviations of the bond angles from the perfect tetrahedral angle of $109.5^{\circ}$, and a small reduction in the occupied valence bandwidth. The EDoS of the Si-Pbam, Si-P $4_{1} 2_{1} 2$, and $\mathrm{Si}-\mathrm{P} 4_{2} / \mathrm{ncm}$ phases are not divided into separate $s$ and $p$ parts, as found in $\mathrm{P} 6522$, see Fig. 2 of Ref. 42, although the overlaps of the $s$ and $p$ parts of the EDoS in the Si structures are not large. The EDoS of Ge-P $4_{1} 2_{1} 2$ is broken into a lower $s$ part and an upper $p$ part, with a small inner gap clearly visible at about $-3.7 \mathrm{eV}$ indicating significant weakening of the $s p$ hybridization. The band structure of Ge-P $4_{2} / \mathrm{ncm}$ is almost broken into $s$ and $p$ parts, while for Ge-Pbam there is significant overlap in the energy scale between both parts, giving a gapless valence EDoS.

\section{VIBRATIONAL PROPERTIES AND DYNAMICAL STABILITY}

The phonon dispersion relations of the Pbam, $\mathrm{P} 4_{1} 2_{1} 2$, and $\mathrm{P} 4_{2} / \mathrm{ncm}$ phases of $\mathrm{Si}$ at zero pressure, calculated using a supercell method with small atomic displacements, ${ }^{52}$ are plotted in Fig. 9, while those of $\mathrm{C}$ and Ge are given in the Supplemental Material. ${ }^{36}$ These plots show that the phases are all dynamically stable at zero pressure. The Pbam phases have distinct and relatively well resolved upper-frequency phonon bands which have rather small dispersion across the Brillouin zone, resulting in a characteristic high-frequency peak in their respective density of states (PhDoS). The phonon band structures and PhDoS of the three phases in Si and Ge phases are quite similar, differing mainly by a scaling arising from the different atomic massses, which reflects the similarity in bonding. For the three materials, the $\mathrm{Ph}-$ DoS of the $\mathrm{P} 4_{2} / \mathrm{ncm}$ phase extends to somewhat lower frequencies than the Pbam phase.

We have also simulated the Raman intensities for the three phases at $300 \mathrm{~K}$, which are shown in Fig. 10. SiPbam has strong high-frequency Raman peaks in the range $450-510 \mathrm{~cm}^{-1}$, and a number of rather weak Raman modes below that frequency range. Its strongest mode lies almost directly on top of the calculated single Raman mode of Si-cd $\left(505 \mathrm{~cm}^{-1}\right.$, cf. the experimental value of $520 \mathrm{~cm}^{-1}$, Ref. 7). This fact is related to the structural similarity of Pbam and diamond discussed previously. In contrast, the strongest Raman peak of Si-P $4_{1} 2_{1} 2$ lies at a frequency of $480 \mathrm{~cm}^{-1}$, somewhat below the diamond mode. Si-P $4_{1} 2_{1} 2$ shows also significant Raman activity just below $200 \mathrm{~cm}^{-1}$ and weak Raman activity around
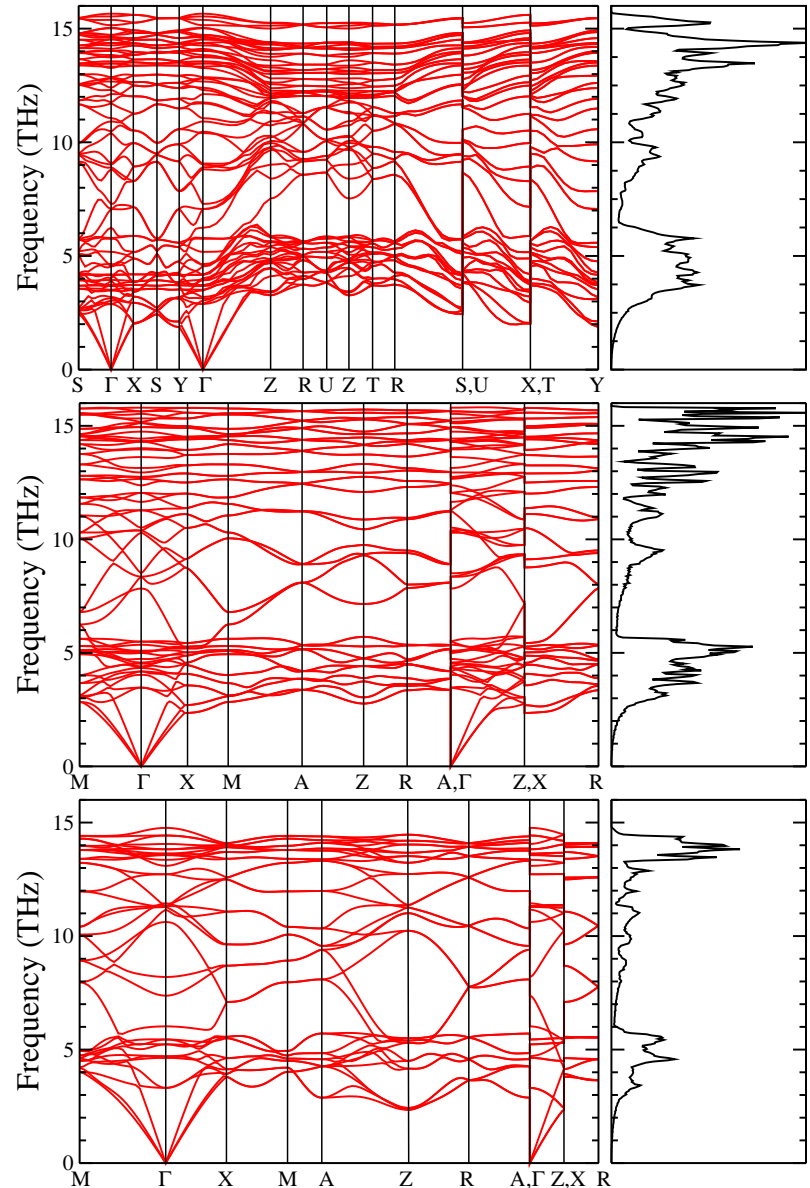

FIG. 9. (Color online) Calculated phonon dispersion relations of the Pbam, $\mathrm{P} 4_{1} 2_{1} 2$, and $\mathrm{P} 4_{2} / \mathrm{ncm}$ structures of $\mathrm{Si}$ at zero pressure. The panel on the right of each plot shows the total phonon density of states.

$330 \mathrm{~cm}^{-1}$, as can be seen in Fig. 10 .

These simulated spectra can be used for the eventual identification of the phases in Raman experiments, although insufficient experimental resolution, broadened peaks, and mixture of phases with overlapping peaks (all of them rather normal situations in nanoindentation or conventional compression experiments) difficult the task of comparison with experimental Raman results. Also, some caution has to be exerted as changing temperature can result in a significant change in the relative intensity of the calculated diffraction peaks. For example, we find significant differences in the relative intensities of the modes reported for $\mathrm{Si}-\mathrm{P} 4_{2} / \mathrm{ncm}$ by Zhao et al. ${ }^{35}$ probably due to the temperature used in simulating the Raman spectra. (Further to this, Zhao et al. used the LDA while the results in Fig. 10 were obtained using the PBE functional.)

Zhao et $a l .{ }^{35}$ also made the interesting proposal that $\mathrm{P} 4_{2} / \mathrm{ncm}$ could explain the experimentally unknown structure of a Si-XIII phase, supported by a comparison of calculated and experimental Raman vibrational data. ${ }^{6,7,21,22}$ Metastable Si-XIII is relevant to thermal 


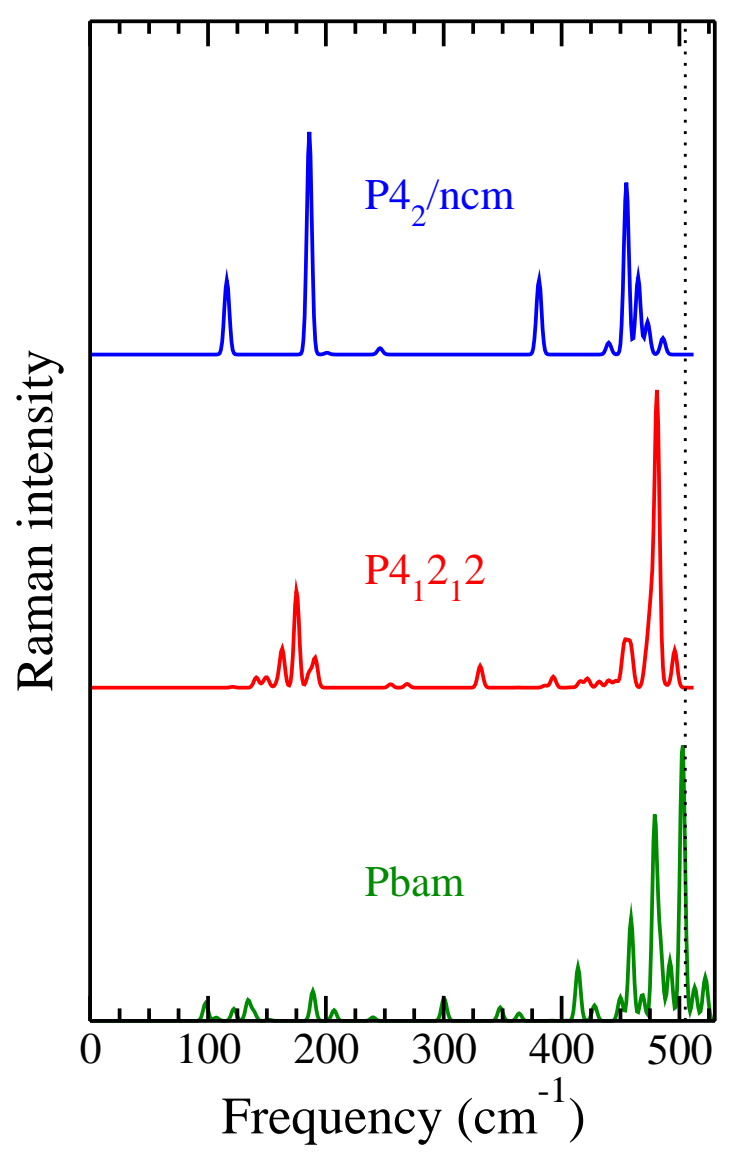

FIG. 10. (Color online) Calculated Raman spectra of the $\mathrm{P} 4_{2} / \mathrm{ncm}, \mathrm{P} 4_{1} 2_{1} 2$, and Pbam structures of $\mathrm{Si}$ at zero pressure and $300 \mathrm{~K}$, calculated within the PBE. The vertical dotted line indicates the calculated frequency of the single intense Raman mode of the Si-cd phase.

processing of silicon wafers, which induces large and potentially damaging stresses. It has been reported in several sources ${ }^{6,7,20-22}$ as being observed only in coexistence with other Si phases (bc8, r8, cd, and hd), which makes it very difficult to determine its structure. The experimental situation concerning Si-XIII has been examined in detail in a recent publication by Ruffell et al. ${ }^{7}$ who have carefully reassessed previous experimental data on this phase. ${ }^{6,20-22}$ These authors conclude the existence of three signature peaks which are characteristics of the Raman spectrum of Si-XIII, at frequencies of 202, 333, and $478 \mathrm{~cm}^{-1}$, which agree quite well with those calculated by us for the $\mathrm{P} 4_{1} 2_{1} 2$ phase (cf. the data in Fig. 10 for Si-P $4_{1} 2_{1} 2$ with those in Fig. 6 of Ref. 7 for the Si-XIII phase). In contrast, the Raman spectrum of the $\mathrm{P} 4_{2} /$ ncm structure, previously suggested for Si-XIII by Zhao et al., ${ }^{35}$ lacks the characteristic peak at $333 \mathrm{~cm}^{-1}$, whereas their calculated peaks at $\sim 110$ and $\sim 380 \mathrm{~cm}^{-1}$ are absent in the experimental data for this phase. We suggest thus that $\mathrm{P} 4_{1} 2_{1} 2$ is a more likely candidate for the structure of the Si-XIII phase.

\section{SUMMARY AND CONCLUSIONS}

Structure searches for group 14 elements using AIRSS have led to the discovery of a number of low-energy structures, including low-density $s p^{3}$ bonded structures of Pbam and $P 4_{1} 2_{1} 2$ symmetries. From the structures that we have studied in C, Si, and Ge, only the cd and hd structures have significantly lower energies than Pbam. Another structure that readily appeared in our searches is the $\mathrm{P} 4_{2} / \mathrm{ncm}$ (or T12) structure previously reported by Zhao et $a l .{ }^{35}$ for which we provide a detailed interpretation in terms of tilting and rebonding of diamond-like tetrahedra, that complements and extends those authors' description. The $\mathrm{P} 4_{2} / \mathrm{ncm}$ structure is the simplest possible that can be built by stacking slabs of tilted tetrahedra while preserving to a large degree a highly regular fourfold coordination for the sites. The Pbam, $\mathrm{P} 4_{1} 2_{1} 2$, and $\mathrm{P} 4_{2} / \mathrm{ncm}$ structures are dynamically stable and have lower energies than a number of metastable polymorphs of group 14 elements that have been synthesized. It is conceivable that one or more of these phases could be formed as metastable phases in indentation or diamond anvil cell experiments, perhaps in combination with heat treatment, and in fact $\mathrm{P} 4_{2} / \mathrm{ncm}$ has been proposed previously as the structure of known yet experimentally unresolved phases of Si and Ge. ${ }^{35}$ We note that an equivalent $\mathrm{P} 4_{2} / \mathrm{ncm}$ structure has recently been suggested to play an important role in the homogeneous crystallization of water. ${ }^{86}$

Our PBE-DFT calculations show semiconducting behavior for the Pbam and $\mathrm{P} 4_{1} 2_{1} 2$ structures at zero pressure, except for Ge-Pbam that is calculated to have a band gap close to zero at low pressures. The band gaps for a particular structure decrease from $\mathrm{C}$ to $\mathrm{Ge}$, as expected. The direct band gap of Si-Pbam at the zone center is estimated to have a value of $1.4 \mathrm{eV}$ (after correcting for the well-known underestimation of the PBEDFT gap) so that it might be suitable for applications in photovoltaics.

We have found several other structures in our searches. These include a low-energy structure of $I 4_{1} / a$ symmetry that is denser than the cd structure in $\mathrm{Si}$ and has a very similar enthalpy to that of the well-known metastable polymorphs $\mathrm{r} 8 / \mathrm{bc} 8$, and which has been suggested to play a role in the decompression kinetics from the high pressure $\beta$-Sn phase. ${ }^{63}$ We also found a low energy and low density structure of $C m c a$ symmetry which, in carbon, is very close in energy to both the Pmmn structure of the so-called $\mathrm{P}$ phase ${ }^{31}$ and the Cmmm structure or Cco-C8 phase, ${ }^{30}$ with which it shares similar structural features. These phases have been proposed for superhard carbon allotropes experimentally obtained after cold compression of carbon nanotubes, ${ }^{27}$ and their closeness suggests that such experimental allotropes could consist of a mixture of energetically and structurally related forms.

Among the proposed structures, the new Pbam polymorph looks particularly promising (low energy, dynam- 
ical stability and direct band gap in $\mathrm{Si}$ ). Although the study of transformation mechanisms between phases and possible synthesis routes is beyond the scope of the present work, its structural features, based on sheared diamond-like slabs, suggests that it might be synthesized by controlled application of uniaxial stress to the diamond phases. (Note added: When this paper was about to be submitted we learnt of very recent findings for Pbam in $\mathrm{C}$ by Baburin et al. ${ }^{87}$ and the synthesis in $\mathrm{Si}$ of an orthorhombic phase with $\mathrm{Cmcm}$ symmetry ${ }^{88}$ and properties quite similar to those that we find for SiPbam. This recent experimental result suggests that the Si-Pbam structure might also be synthesised. Our results for C-Pbam agree with those in Ref. 87 while here we also provide results for $\mathrm{Si}$ and $\mathrm{Ge}^{89}$ )

$\mathrm{P} 4_{1} 2_{1} 2$, a spiral structure with tetragonal symmetry consisting of a packing of pentagonal helices with a large proportion of fivefold rings, is also very interesting and shows several unusual features. It belongs to the same class of chiral frameworks as a previously reported $\mathrm{P}_{5} 22$ structure ${ }^{42}$ and its projection along the four-fold axis yields the so-called Cairo two-dimensional pentagonal tiling. In a very recent publication ${ }^{90}$ that appeared when this paper was about to be submitted, a new two-dimensional carbon allotrope with 24 atoms per cell and $P 42{ }_{1} m$ symmetry was proposed, with exactly the same description in terms of the Cairo tiling as the three-dimensional $\mathrm{P} 4_{1} 2_{1} 2$ structure proposed here. The appearance of the same unusual (yet beautiful) topologi- cal motif in both two- and three-dimensional metastable forms of carbon, obtained independently, is quite interesting and begs for a closer examination of the role of pentagonal rings on the stability of novel and exotic carbon allotropes. The $\mathrm{P} 4_{1} 2_{1} 2$ structure is energetically quite competitive in $\mathrm{Si}$ and $\mathrm{Ge}$, and in $\mathrm{C}$ it has a similar enthalpy to the structures currently proposed for allotropes obtained from compressed nanotubes, ${ }^{27,30,31}$ yet with a very different topology. We find that the $\mathrm{P} 4_{1} 2_{1} 2$ structure shows the best compatibility with the available experimental Raman data for the unknown phase Si-XIII.

We hope that these results will entice experimental searches for new phases of these materials.

\section{ACKNOWLEDGMENTS}

AM acknowledges the financial support of the Ministerio de Educación, Cultura y Deporte (MECD, Spain) through its Programa de Movilidad de Recursos Humanos (Plan Nacional de I+D+i), grant PRX12/00335, and of project MAT2010-21270-C04-03. Access to the MALTA computer cluster (Universidad de Oviedo, Project CSD2007-00045) and the High Performance Computing Service of the University of Cambridge are gratefully acknowledged. RJN and CJP were supported by the Engineering and Physical Sciences Research Council (EPSRC) of the UK. We thank Keith Refson for useful discussions.
1 R. J. Nelmes and M. I. McMahon, Semicond. Semimetals 54, 145 (1998), and references therein.

2 A. Mujica, A. Rubio, A. Muñoz, and R. J. Needs, Rev. Mod. Phys. 75, 863 (2003), and references therein.

${ }^{3}$ C. Frondel and U. B. Marvin, Nature 214, 587-589 (1967).

${ }^{4}$ F. P. Bundy and J. S. Kasper, J. Chem. Phys. 46, 3437 (1967).

${ }^{5}$ R. M. Wentorf and J. S. Kasper, Science 139, 338 (1963).

${ }^{6}$ V. Domnich, Y. Aratyn, W. M. Kriven, and Y. Gogotsi, Rev. Adv. Mater. Sci. 17, 33 (2008).

7 S. Ruffell, B. Haberl, S. Koenig, J. E. Bradby, and J. S. Williams, J. Appl. Phys. 105, 093513 (2009).

${ }^{8}$ F. P. Bundy and J. S. Kasper, Science 139, 340 (1963).

9 J. S. Kasper and S. M. Richards, Acta Crystallogr. 17, 752 (1964).

10 R. O. Piltz, J. R. Maclean, S. J. Clark, G. J. Ackland, P. D. Hatton, and J. Crain, Phys. Rev. B 52, 4072 (1995).

11 B. C. Johnson, B. Haberl, S. Deshmukh, B. D. Malone, M. L. Cohen, J. C. McCallum, J. S. Williams, and J. E. Bradby, Phys. Rev. Lett. 110, 085502 (2013).

12 R. J. Nelmes, M. I. McMahon, N. G. Wright, D. R. Allan, and J. S. Loveday, Phys. Rev. B 48, 9883 (1993).

13 A. Mujica and R. J. Needs, Phys. Rev. B 48, 17010 (1993).

14 R. J. Needs and A. Mujica, Phys. Rev. B 51, 9652 (1995).

15 A. M. Guloy, R. Ramlau, Z. Tang, W. Schnelle, M. Baitinger, and Y. Grin, Nature 443, 320 (2006).

16 J. S. Kasper, P. Hagenmuller, M. Pouchard, and C. Cros,
Science 150, 1713 (1965).

17 A. San-Miguel, P. Kéghélian, X. Blase, P. Mélinon, A. Perez, J. P. Itié, A. Polian, E. Reny, C. Cros, and M. Pouchard, Phys. Rev. Lett. 83, 5290 (1999).

18 M. Beekman and G. S. Nolas, J. Mater. Chem. 18, 842 (2008).

19 Y.-X. Zhao, F. Buehler, J. R. Sites, and I. L. Spain, Solid State Commun. 59, 679 (1986).

20 A. Kailer, Y. G. Gogotsi, and K. G. Nickel, J. Appl. Phys. 81, 3057 (1997).

21 V. Domnich and Y. Gogotsi, Rev. Adv. Mater. Sci. 3, 1 (2002).

22 D. Ge, V. Domnich, and Y. Gogotsi, J. Appl. Phys. 95, 2725 (2004).

23 A. K. Geim and K. S. Novoselov, Nature Materials 6, 183 (2007).

24 W. L. Mao, H.-k. Mao, P. J. Eng, T. P. Trainor, M. Newville, C.-c. Kao, D. L. Heinz, J. Shu, Y. Meng, R. J. Hemley, Science 302, 425 (2003).

25 S. Yamanaka, A. Kubo, K. Komaguchi, N. S. Kini, T. Inoue, and T. Irifune, Phys. Rev. Lett. 96, 076602 (2006).

26 M. Popov, M. Kyotani, R. J. Nemanich, and Y. Koga, Phys. Rev. B 65, 033408 (2002).

27 Z. Wang, Y, Zhao, K. Tait, X. Liao, D. Schiferl, C. Zha, R. T. Downs, J. Qian, Y. Zhu, and T. Shen, Proc. Natl. Acad. Sci. U.S.A. 101, 136998 (2004).

${ }^{28}$ Q. Li, Y. Ma, A. R. Oganov, H. Wang, H. Wang, Y. Xu, T. 
Cui, H.-k. Mao, and G. Zou, Phys. Rev. Lett. 102, 175506 (2009).

29 A. O. Lyakhov and A. R. Oganov, Phys. Rev. B 84, 092103 (2011).

30 Z. Zhao, B. Xu, X.-F. Zhou, L.-M. Wang, B. Wen, J. He, Z. Liu, H.-T. Wang, and Y. Tian, Phys. Rev. Lett. 107, $215502(2011)$

31 H. Niu, X.-Q. Chen, S. Wang, D. Li, W. L. Mao, and Y. Li, Phys. Rev. Lett. 108, 135501 (2012).

32 Q. Zhu, A. R. Oganov, M. A. Salvadó, P. Pertierra, and A. O. Lyakhov, Phys. Rev. B 83, 193410 (2011)

33 S. Botti, J. A. Flores-Livas, M. Amsler, S. Goedecker, and M. A. L. Marques, Phys. Rev. B 86, 121204 (2012).

${ }^{34}$ H. J. Xiang, B. Huang, E. Kan, S.-H. Wei, and X. G. Gong, Phys. Rev. Lett. 110, 118702 (2013).

35 Z. Zhao, F. Tian, X. Dong, Q. Li, Q. Wang, H. Wang, X. Zhong, B. Xu, D. Yu, J. He, H.-T. Wang, Y. Ma, and Y. Tian, J. Am. Chem. Soc. 134, 12362 (2012).

36 See Supplemental Material at http://link.aps.org/supplemental/xxxxxxx/yyyyyyyyy for more details of the structures, electronic band structures, and densities of states.

37 C. J. Pickard and R. J. Needs, Phys. Rev. Lett. 97, 045504 (2006).

38 C. J. Pickard and R. J. Needs, J. Phys.: Condensed Matter 23, 053201 (2011).

39 C. J. Pickard and R. J. Needs, Phys. Rev. B 76, 144114 (2007).

40 G. Csanyi, C. J. Pickard, B. D. Simons, and R. J. Needs, Phys. Rev. B 75, 085432 (2007).

41 A. J. Morris, C. J. Pickard, and R. J. Needs, Phys. Rev. B 80, 144112 (2009)

42 C. J. Pickard and R. J. Needs, Phys. Rev. B 81, 014106 (2010).

43 J. Sun, D. D. Klug, C. J. Pickard, and R. J. Needs, Phys. Rev. Lett. 106, 145502 (2011).

${ }^{44}$ M. Martinez-Canales, C. J. Pickard, and R. J. Needs, Phys. Rev. Lett. 108, 045704 (2012).

45 S. J. Clark, M. D. Segall, C. J. Pickard, P. J. Hasnip, M. I. J. Probert, K. Refson, and M. C. Payne, Z. Kristallogr. 220, 567 (2005).

46 J. P. Perdew, K. Burke, and M. Ernzerhof, Phys. Rev. Lett. 77, 3865 (1996).

47 D. Vanderbilt, Phys. Rev. B 41, 7892 (1990).

${ }^{48}$ G. Kresse and J. Hafner, Phys. Rev. B 47, 558 (1993).

49 G. Kresse and J. Furthmüller, Comput. Mater. Sci. 6, 15 (1996); Phys. Rev. B 54, 11169 (1996). For more information see: http://cms.mpi.univie.ac.at/vasp.

50 P. E. Blöchl, Phys. Rev. B 50, 17953 (1994).

51 G. Kresse and D. Joubert, Phys. Rev. B 59, 1758 (1999).

52 D. Alfè, Computer Physics Communications 180, 26222633 (2009)

53 D. M. Ceperley and B. J. Alder, Phys. Rev. Lett. 45, 566 (1980), as parametrized by J. P. Perdew and A. Zunger, Phys. Rev. B 23, 5048 (1981).

54 J. P. Perdew, A. Ruzsinszky, G. I. Csonka, O. A. Vydrov, G. E. Scuseria, L. A. Constantin, X. Zhou, and K. Burke, Phys. Rev. Lett. 100, 136406 (2008).

55 F. Birch, Phys. Rev. 71, 809 (1947).

${ }^{56}$ R. R. Reeber and K. Wang, J. Electron. Mater. 25, 63 (1996).

57 F. Occelli, P. Loubeyre, and R. LeToullec, Nat. Mater. 2, $151(2003)$

58 J. S. Tse and W. B. Holzapfel, J. Appl. Phys. 104, 043525
(2008).

59 K. Kunc, I. Loa, and K. Syassen, Phys. Rev. B 68, 094107 (2003).

60 R. Maezono, A. Ma, M. D. Towler, and R. J. Needs, Phys. Rev. Lett. 98, 025701 (2007).

${ }^{61}$ R. J. Needs, M. D. Towler, N. D. Drummond, and P. López Ríos, J. Phys.: Condensed Matter 22, 023201 (2010).

${ }^{62}$ We have also obtained several other diamond-like polytypes lying at energies similar to cubic and hexagonal diamond.

63 J.-T. Wang, C. Chen, H. Mizuseki, and Y. Kawazoe, Phys. Rev. Lett. 110, 165503 (2013).

64 B. D. Malone and M. L. Cohen, Phys. Rev. B 85, 024116 (2012).

65 B. D. Malone and M. L. Cohen, Phys. Rev. B 86, 054101 (2012).

66 D. Selli, I. A. Baburin, R. Martonak, and S. Leoni, Scientific Reports 3, 1466 (2013).

67 A. Mujica, S. Radescu, A. Muñoz, and R. J. Needs, Phys. Status Solidi b, 223, 379 (2001).

68 A. Mujica, A. Muñoz, and R. J. Needs, Phys. Rev. B 57, 1344 (1998); A. Mujica, A. Muñoz, S. Radescu, and R. J. Needs, Phys. Status Solidi b, 211, 345 (1999).

69 Q. Zhu, Q. Zeng, and A. R. Oganov, Phys. Rev. B 85, 201407 (2012).

70 Y. Saito, S. Yatsuya, K. Mihama, and R. Uyeda, Jpn. J. Appl. Phys. 17, 291 (1978); Y. Saito, J. Cryst. Growth 47, 61 (1979).

71 A second and denser state with the same $P 4_{2} / \mathrm{ncm}$ symmetry but lying higher in enthalpy has also appeared in our survey.

72 S. Botti, M. Amsler, J. A. Flores-Livas, P. Ceria, S. Goedecker, and M. A. L. Marques, Phys. Rev. B 88, 014102 (2013).

73 These low-symmetry structures, named 20A, 22A, and 24A in Ref. 72 , did not appear in our searches.

74 N. Rey, A. Muñoz, P. Rodríguez-Hernández, A. San Miguel, J. Phys.: Condens. Matter 20, 215218 (2008).

75 J. Dong and O. F. Sankey, J. Phys.: Condens. Matt. 11, 6129 (1999).

76 C. W. Myles, J. Dong, and O. F. Sankey, Phys. Rev. B 64, $165202(2001)$

77 A. J. Karttunen, T. F. Fässler, M. Linnolahti, and T. A. Pakkanen, Inorg. Chem. 50, 1733 (2011).

${ }^{78}$ G. B. Adams, M. O'Keeffe, A. A. Demkov, O. F. Sankey, and Y.-M. Huang, Phys. Rev. B 49, 8048 (1994).

79 J. Rodriguez-Carvajal, Physica B 192, 55 (1993). The complete program and documentation can be obtained at http://www.ill.eu/sites/fullprof/

80 For clarity, a lighter colour hue has been used for the corners and bonds of the upper slab (which are equivalent to those in the lower slab).

${ }^{81}$ In their description of the structure, Zhao et al. ${ }^{35}$ reported it as having five different angles, but in fact there are six. Our calculated values are: $103.0^{\circ}, 105.7^{\circ}, 108.3^{\circ}, 109.1^{\circ}$, $111.8^{\circ}$, and $122.5^{\circ}$.

82 These are not planar rings: one half (blue) of each ring is in one plane and the other (red) in another plane a distance c/2 apart.

83 The same $\mathrm{P} 6522$ structure was later obtained by Lyakhov et $a l .{ }^{29}$ using related structure-searching methods.

84 M. Gardner, Scientific American 233, 112 (1975).

85 I. Jimenez, L. J. Terminello, D. G. J. Sutherland, J. A. Carlisle, E. L. Shirley, and F. J. Himpsel, Phys. Rev. B 
56, 7215 (1997).

86 J. Russo, F. Romano, and H. Tamaka, Nature Materials 13, 733 (2014)

87 I.A. Baburin, D.M. Proserpio, V.A. Saleev, and A.V. Shipilova, Phys. Chem. Chem. Phys. 17, 1332 (2015).

88 D. Y. Kim, S. Stefanoski, O. O. Kurakevych, and T. A. Strobel, Nature Materials 14, 169 (2015).

89 We presented the main results of our study, including de- scriptions of the new Pbam and $\mathrm{P} 4_{1} 2_{1} 2$ structures, at the EHPRG52 Conference, focusing on $\mathrm{Si}$ and stating that essentially the same qualitative results were obtained for $\mathrm{C}$ and Ge. A scientific report related to the grant PRX12/00335 had been previously submitted by one of us (AM) to the Spanish MECD.

90 S. Zhang, J. Zhou, Q. Wang, X. Chen, Y. Kawazoe, and P. Jena, Proc. Natl. Acad. Sci. U.S.A. 112, 2372 (2015). 